\title{
Gut microbiota and central nervous system condemned to understand each other: their role in multiple sclerosis
}

\section{Introduction}

The possible relationship between microbiota and inflammatory processes has been known since the 1990's. However, it was at the beginning of the $21^{\text {st }}$ century that the role played by commensal bacteria (normal microflora, indigenous microbiota) of the gastrointestinal tract in immunomodulating phenomena began to be examined, and, therefore, its effects on the pathogenesis of chronic inflammation, such as ulcerative colitis, rheumatoid arthritis, Crohn disease, arteriosclerosis, colon cáncer, metabolic diseases and others. Thus, the data show how gut microbiota modulates the production of proinflammatory cytokines such as TNF- $\alpha$. However, it can be said that it has been in the last five years when studies made have evidenced a possible relationship between the central nervous system (CNS) and gut tract microbiota, or, similarly, the possible intervention of gut microbiota in neuroinflammatory and neurogenerative processes such as depresion, autism, cognitive disorders (dementia), etc. and, possibly, multiple sclerosis. ${ }^{1,2}$

Gut microbiota is essential in mammals for the correct development of gut flora and the maturation of the host's immune system, generating a complex and delicate balance called "microbioma", whose alteration triggers a dysregulation of the immune response, which ends up exerting effects on the gut tract and CNS. ${ }^{1,3-7}$ However, the mechanisms on which these effects and relationships are established have not currently been clarified.

The term microbiota refers to the commensal microorganisms (of the trillion of these existing on external and internal surfaces of our organism) resident in a specific habitat of our body. Of these, the gastrointestinal tract presents from $10^{13}-10^{14}$ resident bacteria, which respond to diet, obesity, immune system dysfunction, environmental factors, leaky gut, psychosocial stress, early life stress and drug consumption. ${ }^{3,8,9}$

Disbiosis (or disbacteriosis) can be generated by a bad understanding between host and microbiota, and, specifically, between the receptors of the former and the ligands derived from the latter. Any of the metabolites produced by the bacterial flora (fermentation products, neuroactive molecules or bacterial immunostimulators like lipopolysaccharides), could cause immunological or even neuropsychiatric disorders. ${ }^{3}$ The capacity of certain bacterial antigens such as lipopolysaccharide A to mediate in the traffic and migration of a population of intestinal antigen-presenting cells to the lymphoid tissue, suggests an important cross-talk between the intestinal mucous and the brain/spinal cord. ${ }^{1}$

Recent data point to the possibility that the alteration in the dialogue between intestinal microbiota-CNS and immune system dysregulation may play an important role in the pathogenesis of multiple sclerosis (MS). ${ }^{1}$ For instance; elevations in the levels of antibodies against gastrointestinal antigens in patients with MS have been observed. ${ }^{10}$
Volume I Issue I - 2014

\author{
Begoña Escribano,' Isaac Túnez \\ 'Department of Cell Biology, Physiology and Immunology, \\ University of Cordoba, Spain \\ 2Department of Biochemistry and Molecular Biology, IMIBIC/ \\ University of Cordoba, Spain
}

\begin{abstract}
Correspondence: Isaac Tunez, Department of Biochemistry and Molecular Biology, Faculty of Medicine, Maimonides Institute for Research in Biomedicine of Cordoba (IMIBIC)/Reina Sofia University Hospital/University of Cordoba,Av. Menendez Pidal $\mathrm{s} / \mathrm{n}$, University of Cordoba I4004, Spain, Tel +342 18268, Fax +34957218229, Email fm2tufii@uco.es
\end{abstract}

Received: May 29, 2014 | Published: May 30, 2014

MS is the main cause of invalidating non-traumatic neurodegenerative disease of a neuroinflammatory and demyelinating nature in young adults. Of an unknown etiology, genetic, infectious, environmental, geographic, etc. factors have been involved in its etiopathogenia. $^{11-13}$

On these lines, the studies made on the model of experimental autoimmune encephalomyelitis (EAE) suggest that certain intestinal bacterial populations lead to proinflammatory conditions, which result in the onset of autoimmune diseases, among which is multiple sclerosis. ${ }^{1}$ Thus, the sterilization of the intestine with antibiotics reduces the severity of EAE. ${ }^{3}$ All this is due to the attenuation of the proinflammatory response of the $T_{H} 1, T_{H} 17$ cells, T-regulating cells, invariant natural killers or $\mathrm{T}$ and $\mathrm{B}$ cells, whereas colonization by segmented filamentous bacteria (SFB) makes them more sensitive. ${ }^{3}$ On the other hand, Wang and Kasper showed how the oral administration of different types of bacteria or mixtures of these can trigger either a reduction or an exacerbation of the symptoms manifested in EAE, depending on the type of bacteria. ${ }^{9}$

Some data supplied by our group in patients with relapsingremitting MS (RRMS) recently showed that the status of the patient's disease was associated with changes in oxidative damage biomarkers and antioxidant systems at a peripheric level and that these values returned to normal in patients submitted to treatment with Natalizumab (a monoclonal antibody used in MS treatment; anti-VLA-4; Tysabri ${ }^{\circledR}$, Biogen Idec, Cambridge, MA, USA). ${ }^{14-16}$ This situation has been supported and verified by data, not yet published, of our group in the animal model of EAE induced by the myelin oligodendrocyte glycoprotein (MOG).

In this study, in which 2-month old Dark Agouti rats with an average weight of $190 \mathrm{~g}$ were used, it was seen that the administration of MOG and the development of EAE was associated with the release of lipopolysaccharide A (LPS), probably due to changes in the gastrointestinal microbiota characterized by rises in its levels in blood and CNS. These increases were correlated with the severity 
of the process suffered by the animals. Furthermore, the rats with EAE treated with Natalizumab or dimethyl fumarate (Nrf2 inductor) underwent a significant improvement in their clinical score associated with a reduction in circulating and tissue levels LPS. All this appeared as being correlated with the levels of oxidative damage (evaluated as changes in levels of lipid peroxidation products, carbonylated proteins, reduced glutathione (GSH) and GSH/oxidized glutathione ratio $(\mathrm{GSH} / \mathrm{GSSG})$

The results indicate a close relationship between microbiota and oxidative stress in EAE, and probably in MS, and its likely key role, at least, in the pathogenesis and evolution of these processes. However, more information and studies are necessary to reaffirm the concept of a microbioma-intestine-brain axis in the pathogenesis of demyelinating autoimmune diseases.

\section{Acknowledgements}

None.

\section{Conflict of interest}

The author declares no conflict of interest.

\section{References}

1. Ochoa-Reparaz J, Mielcarz DW, Begum-Haque S, et al. Gut, bugs, and brain: role of commensal bacteria in the control of central nervous system disease. Ann Neurol. 2011;69(2):240-247.

2. Tlaskalova-Hogenova H, Stepankova R, Hudcovic T, et al. Commensal bacteria (normal microflora), mucosal immunity and chronic inflammatory and autoimmune diseases. Immunol Lett. 2004;93(23):97-108.

3. Berer K, Krishnamoorthy G. Commensal gut flora and brain autoimmunity: a love or hate affair? Acta Neuropathol. 2012;123(5):639651 .

4. Ehlers S, Kaufmann SH. Infection, inflammation, and chronic diseases: consequences of a modern lifestyle. Trends Immunol. 2010;31(5):184 190 .
5. Ott SJ, Schreiber S. Reduced microbial diversity in inflammatory bowel diseases. Gut. 2006;55(8):1207.

6. Turnbaugh PJ, Hamady M, Yatsunenko T, et al. A core gut microbiome in obese and lean twins. Nature. 2009;457(7228):480-484.

7. Turnbaugh PJ, Ley RE, Mahowald MA, et al. An obesity-associated gut microbiome with increased capacity for energy harvest. Nature. 2006;444(7122):1027-1031.

8. Haroon E, Raison CL, Miller AH. Psychoneuroimmunology meets neuropsychopharmacology: translational implications of the impact of inflammation on behavior. Neuropsychopharmacology. 2012;37(1):137162.

9. Wang Y, Kasper LH. The role of microbiome in central nervous system disorders. Brain Behav Immun. 2014;38:1-12.

10. Wekerle H, Berer K, Krishnamoorthy G. Remote control-triggering of brain autoimmune disease in the gut. Curr Opin Immunol. 2013;25(6):683-689.

11. Lassmann H, Bruck W, Lucchinetti CF. The immunopathology of multiple sclerosis: an overview. Brain Pathol. 2007;17(2):210-218.

12. Lassmann H, Van Horssen J. The molecular basis of neurodegeneration in multiple sclerosis. FEBS Lett. 2011;585(23):3715-3723.

13. Lassmann H, Van Horssen J, Mahad D. Progressive multiple sclerosis: pathology and pathogenesis. Nat Rev Neurol. 2012;8(11):647-656.

14. Tasset I, Agüera E, Gascon F, et al. [Natalizumab and reduction of carbonylated proteins in patients with multiple sclerosis]. Rev Neurol. 2012;54(8):449-452.

15. Tasset I, Agüera E, Sanchez-Lopez F, et al. Peripheral oxidative stress in relapsing-remitting multiple sclerosis. Clin Biochem. 2012;45(6):440 444.

16. Tasset I, Bahamonde C, Agüera E, et al. Effect of natalizumab on oxidative damage biomarkers in relapsing-remitting multiple sclerosis. Pharmacol Rep. 2013;65(3):624-631. 